\title{
Untersuchungen zur Bestimmung der 4-Hydroxy-3-methoxy-mandelsäure (Vanillinmandelsäure) im Urin
}

\author{
Von H. WISSER und D. STAMM \\ Aus dem Max-Planck-Institut für Psychiatrie, München
}

(Eingegangen am 7. August 1969)

Das Verfahten zur Bestimmung der 4-Hydroxy-3-methoxy-mandelsäure im Urin nach Prsano, Crout und Abraham (1) wurde modifiziert. Einige Parameter der Methode, wie Menge des Oxydationsmittels, Reaktionszeit, Reaktionstemperatur und der Einfluß der Urinlcerwerte, wurden untersucht. Für ein Kollektiv von 51 Männern und 47 Frauen wird der Normalbereich für die 24-Stunden-Ausscheidung angegeben. Die Alters- und Geschlechtsabhängigkeit wurde geprüft. Die Methode wurde angewandt bei der Analyse von 3-Stunden-Urinproben.

\section{Studies on the measurement of 4-bydroxy-3-methoxy-mandelic acid (vanillinmandelic acid) in urine}

The method of Pisano, CRout and Abraham (1) for the measurement of urinary 4-hydroxy-3-methoxy-mandelic acid was modified. Certain parameters of the method, like the amount of oxidising agent, reaction time, reaction temperature and the influence of the blank urinary values were studied. The normal range for the 24 hour excretion is given for a collective of 51 men and 47 vomen. Age and sex-dependence were tested. The method was used for the analysis of 3-hour urine samples.

Die 4-Hydroxy-3-methoxy-mandelsäure (Vanillinmandelsäure) ist beim Menschen das Hauptabbauprodukt von Adrenalin und Noradrenalin. Ihre Ausscheidung im 24-Stunden-Urin liegt im mg-Bereich und ist etwa hundertfach größer als die Summe der sogenannten Katecholamine. Dies ermöglicht die Bestimmung der Vanillinmandelsäure im Urin mit Methoden, die nicht mit der Störanfälligkeit der Fluorometrie, die bei der Bestimmung der Katecholamine angewandt wird, behaftet sind.

Bei der Bestimmung der Vanillinmandelsäure im Urin wurden Papier- $(2,3)$, Dünnschicht- $(4,5,6)$ und Säulenchromatographie $(7,8)$, Hochspannungselektrophorese (9) und flüssig-flüssig-Verteilungen (1, 10, 11) einzeln oder in Kombination als Trennverfahren benutzt. Bei den ersten vier ist eine mehr oder minder vollständige Trennung der. verschiedenen Komponenten möglich, so daß eine unspezifische Bestimmungsmethode, wie die Kupplung mit diazotiertem $p$-Nitroanilin $(4,7,8)$ oder $p$-Aminophenyl- $\beta$-diäthylaminoäthylsulfon $(6,12)$ angewandt werden kann. Am häufigsten wird zur Abtrennung der Vanillinmandelsäure das Ausschütteln des angesäuerten Urins mit Äthylacetat benutzt. Bei dieser Gruppentrennung braucht man aber ein spezifisches Bestimmungsverfahren. Die notwendige Spezifität erhält man dadurch, $\mathrm{daB}$ die Vanillinmandelsäure oxydativ mit Natriummeta-perjodat (1), Kaliumferricyanid (10), Kupferionen (11), durch katalytische Oxydation unter Druck (13) oder fermentativ (14) in Vanillin überführt wird. Die Bestimmung des aus neutraler Lösung isolierten $\mathrm{Va}$ nillins ist auf verschiedene Weise möglich, einmal durch Messung der Extinktion des Vanillins in alkalischer Lösung bei $360 \mathrm{~nm}$ oder nach Umsatz mit Indol bei $495 \mathrm{~nm}$. Von Wirk und Mitarbeitern (15) wurde
Vanillin nach Umsatz mit Trifluoroacetanhydrid gaschromatographisch bestimmt. Als weitere Verfahren der Vanillinmandelsäure-Bestimmung sei die Isotopenverdünnungstechnik genannt (8).

Sieht man von speziellen Fragestellungen $a b$, so dürfte die Methode „Abtrennung der Vanillinmandelsäure durch Ausschütteln mit Äthylacetat und Natrium-metaperjodat oder Kaliumferricyanid als Oxydationsmittel ${ }^{\text {cc }}$ allgemeine Anwendung finden. Die Vanillinbildung mit Kupferionen hat den Nachteil, daß ein Teil der Homovanillinsäure in Vanillin überführt wird (16). Die gaschromatographische Bestimmung dürfte wegen ihrer Aufwendigkeit nur bei besonderen Fragestellungen Anwendung finden. Sie hat den Vorteil, daß mehrere Bestandteile gleichzeitig erfaßt werden.

\section{Methodik}

Die im folgenden beschriebene Bestimmung lehnt sich eng an das von J. J. PISANO und Mitarbeitern (1) beschriebene Verfahren an. Dabei wird die Vanillinmandelsäure durch Ausschütteln des angesäuerten Urins mit Athylacetat abgetrennt. Durch Oxydation mit Natrium-meta-perjodat wird die Vanillinmandelsäure in Vanillin überführt. Die Extinktion des isolierten Vanillins wird in alkalischet Lösung bei $360 \mathrm{~nm}$ bestimmt.

Die Vorschrift (1) wurde dahingehend modifiziert, daß die organischen Phasen nicht aliquotiert werden. Dies hat den Vorteil, $\mathrm{da} ß$ die organischen Lösungsmittel nicht genau abpipettiert werden müssen. Zum anderen braucht auf Verluste durch Verdunsten während der Analyse nicht besonders geachtet zu werden. Allerdings ist es wichtig, daß die Unterphasen - falls gefordert vollständig entfernt werden. Außerdem wurde die Durchführung so geändert, daß für eine Bestimmung mit Leerwert nicht mehr sieben Schüttelgefäße, wie in der Originalvorschrift, sondern drei Schüttelgefäße notwendig sind.

\section{Apparate und Glasgeräte}

1. $50 \mathrm{~m} /$ Zentrifugengläscr, $11 \mathrm{~cm}$ hoch, mit NS 29 , die unten spitz ausgezogen sind. 
2. Quickfit - Austeilautomaten für die organischen Lösungsmittel ${ }^{\mathbf{y}}$ ).

3. Schüttelmaschine $\left.{ }^{2}\right)$.

4. Wasserbad $-50^{\circ}$.

5. $20 \mathrm{ml}$ Rekordspritze mit abgestumpfter Nadel.

6. $\mathrm{MT}-4$ Küvetten, $1 \mathrm{~cm}$ Schichtdicke; Spektralphotometer PMQ $1 I^{3}$ ).

\section{Reagenzien}

Wenn nicht besonders vermetkt, wurden p. a. Reagenzien der Firma Merck, Darmstadt, benutzt.

1. $\mathrm{NaJO}_{4}, 2$ proz. in Wasser (stabil 1 Woche bei $25^{\circ}$ )

2. $\mathrm{Na}_{2} \mathrm{~S}_{2} \mathrm{O}_{5}, 10$ proz. in Wasser (stabil 1 Woche bei $3^{\circ}$ )

3. $1 \mathrm{M} \mathrm{K}_{2} \mathrm{CO}_{3}$-Lösung

4. $5 \mathrm{~N}$ Essigsäure

5. $2 \mathrm{~N} \mathrm{KH}_{2} \mathrm{PO}_{4}$ mit $7 \mathrm{~N} \mathrm{NaOH}$ auf $\mathrm{pH} \mathrm{7,5} \mathrm{eingestellt.}$

(2N $\mathrm{KH}_{2} \mathrm{PO}_{4}$ in einem 1-Liter-Meßkolben mit etwa $850 \mathrm{~m}$ l demin. Wasser gelöst und dann mit $7 \mathrm{~N} \mathrm{NaOH}$ auf $\mathrm{pH} 7,5$ eingestellt. Volumen mit demin. Wasser auf 1 Liter aufgefüllt)

6. Äthylacetat p. a.

7. Toluol p. a.

8. $\mathrm{NaCl}$ p.a.

9. Vanillinmandelsäure-Standard

Stammlösung: $40 \mathrm{mg}$ Vanillinmandelsäure ${ }^{4}$ ) pro $10 \mathrm{ml} 0,01 \mathrm{~N} \mathrm{HCl}$ Arbeitslösung: 1:10 Verdünnung mit demin. Wasser

Von dieser Arbeitslösung werden jeweils $100 \mu l$ für einen primären Standard entnommen.

\section{Arbeitsweise}

$5 \mathrm{~m} l$ Urin werden in ein $50 \mathrm{~m} l$ Zentrifugenglas pipettiert und mit $0,5 \mathrm{ml} 6 \mathrm{~N} \mathrm{HCl}$ angesäuert. Dieser Lösung wird $1 \mathrm{~g} \mathrm{NaCl}$ zugesetzt. Der so vorbereitete Urin wird anschließend mit $30 \mathrm{ml}$ Äthylacetat $5 \mathrm{Min}$. geschüttelt. Zur sauberen Phasentrennung wird das Gemisch $5 \mathrm{Min}$. bei etwa $2000 \mathrm{U}$./Min. zentrifugiert. (Die Bedingungen für das Ausschütteln und Zentrifugieren sind im weiteren Verlauf der Analyse immer gleich.) Die Unterphase wird anschließend mit einer Rekordspritze abgezogen. Das Abziehen der Unterphase muß sorgfältig durchgeführt werden. Nach Abziehen der Unterphase empfiehlt es sich, die Gläser noch einmal $1 \mathrm{Min}$. zu zentrifugieren, um die letzten Reste zu entfernen. Das verbleibende Äthylacetat wird nun in dem gleichen Gefäß mit $3,0 \mathrm{~m} / 1 \mathrm{M}_{2} \quad \mathrm{~K}_{2} \mathrm{CO}_{3}$-Lösung geschüttelt und zentrifugiert. Anschließend wird das Äthylacetat weitgehend abgesaugt.

Je $1 \mathrm{~m} /$ det $\mathrm{K}_{2} \mathrm{CO}_{3}$-Lösung werden in zwei neue Zentrifugengläser pipettiert. Die Probe wird mit $0,2 \mathrm{ml} 2$ proz. $\mathrm{NaJO}_{4}-\mathrm{Lö-}$ sung versetzt und $30 \mathrm{Min}$. bei $50^{\circ}$ erwärmt. Der Leerwert wird ohne Zusatz des Oxydationsmittels analog behandelt. Anschließend werden die Proben abgekühlt und mit $0,2 \mathrm{ml} 10$ proz. $\mathrm{Na}_{2} \mathrm{~S}_{2} \mathrm{O}_{5}$ Lösung und $0,3 \mathrm{~m} l 5 \mathrm{~N}$ Essigsäure versetzt. Nach Zugabe der Essigsäure wird das Probegefäß geschüttelt, wobei $\mathrm{CO}_{2}$ entweicht. Dann gibt man zu der Lösung $0,6 \mathrm{~m} / 2 \mathrm{~N}$ Phosphatpuffer, $\mathrm{pH} 7,5$. Das Gemisch, dessen pH-Wert nun 7,5-7,6 beträgt, wird mit $30 \mathrm{ml}$ Toluol ausgeschüttelt. Der Leerwert wird nach dem Abkühlen zuerst mit $0,2 \mathrm{ml} 10$ proz. $\mathrm{Na}_{2} \mathrm{~S}_{2} \mathrm{O}_{5}$-Lösung versetzt, geschüttelt und dann mit $0,2 \mathrm{~m} / 2$ proz. $\mathrm{NaJO}_{4}$-Lösung. Die Reihenfolge Reduktionsmittel und dann Oxydationsmittel ist einzuhalten. Die Einstellung des pH-Wertes und das Ausschütteln mit Toluol erfolgt in der gleichen Weise wie bei den Proben. Im weiteren werden Probe und Leerwert wieder gleich behandelt.

1) Quickfit Laborglas GmbH., Wiesbaden-Schierstein.

2) Schüttelmaschine: Die benutzte Schüttelmaschine wurde in Zusammenarbeit mit Herm P. Doerr und der mechanischen Werkstatt des Instituts gebaut. Sie ist so ausgelegt, daß 20 bis 36 Gläser unterschiedlichen Durchmessers mittels einer Halteschiene an einem Balken befestigt werden. Die Schüttelgefäße werden mit einem Kunststoffstopfen ohne weitere Sicherung verschlossen. Selbst bei Verwendung eines niedrig siedenden Lösungsmittels ist diese Art des Verschlusses ausreichend. Anschließend wërden die Proben durch Drehen des Balkens geschüttelt.

3) Carl Zeiss, Oberkochen/Württemberg.

4) Serva Entwicklungslabor, Heidelberg.
Nach dem Ausschütteln mit Toluol wird die wäßr. Phase nach Phasentrennung mit einer Rekordspritze abgezogen.

Dies muß nicht quantitativ erfolgen; denn die Toluolphase wird nochmals mit $2,0 \mathrm{ml}$ Phosphatpuffer $\mathrm{pH}$ 7,5 gewaschen. Zur sauberen Phasentrennung wird erneut zentrifugiert und anschlieBend die Unterphase entfernt. Dieser letztere Vorgang wird wiederholt, um Reste der wäßr. Phase vollständig zu entfernen. Das Toluol wird nun mit $2,0 \mathrm{ml} 1 \mathrm{M} \mathrm{K}_{2} \mathrm{CO}_{3}$-Lösung ausgeschüttelt und erneut unter den oben angegebenen Bedingungen zentrifugiert. Die Oberphase wird weitgehend abgezogen und die Unterphase mit einer Pipette in ein Reaktionsgefä $B$ Eppendorf überführt. Die Extinktion dieser $\mathrm{K}_{2} \mathrm{CO}_{3}$-Lösung wird bei $360 \mathrm{~nm}$ gegen $1 \mathrm{M} \mathrm{K} \mathrm{K}_{2} \mathrm{CO}_{3}$ als Leerwert gemessen.

Meßbedingungen am PMQ II

Verstärkung: $10 / \mathrm{I} / \mathrm{I}$

Bandbreite: $0,4-0,5 \mathrm{~nm}$

Falschlicht-Schutzfilter: $320-380 \mathrm{~nm}$

\section{Standard}

Die Auswertung erfolgt über drei Vanillinmandelsäurestandards, die durch die ganze Analyse mitgefühtt werden, wobei $100 \mu l$ Arbeitslösung in $5 \mathrm{ml}$ demin. Wasser nach Zusatz von $0,5 \mathrm{ml}$ 6N $\mathrm{HCl}$ und $1 \mathrm{~g} \mathrm{Kochsalz} \mathrm{mit} \mathrm{der} \mathrm{entsprechenden} \mathrm{Menge} \mathrm{Äthyl-}$ acetat ausgeschüttelt werden. Die weitere Aufarbeitung erfolgt wie oben beschrieben.

\section{Berecbnung}

Der Vanillinmandelsäure-(VMS)-Gehalt einer Probe errechnet sich auf folgende Weise:

$$
\begin{array}{ll}
\qquad \mathrm{g} \mathrm{VMS}=\left(\mathrm{E}_{\mathrm{P}}-\mathrm{E}_{\mathrm{PL}}\right) \frac{\mu \mathrm{g} \mathrm{VMS}}{\mathrm{E}_{\mathrm{S}}-\mathrm{E}_{\mathrm{SL}}} \\
\mathrm{E}=\text { Extinktion } & \mathrm{S}=\text { Standard } \\
\text { Indices: } & \mathrm{PL}=\text { Probenleerwert } \\
\mathrm{P}=\text { Probe } & \mathrm{SL}=\text { Standardleerwert. }
\end{array}
$$

\section{Erläuterungen}

Beim Ansetzen der Urinleerwerte sollte man die angegebene Reihenfolge der Zugabe von Reduktions- und Oxydationsmittel einhalten. Nicht eigens aufgeführte Versuche ergaben, daß bei reinen Lösungen schon eine Einwirkung des Oxydationsmittels von 1-2 Min. bei Zimmertemperatur genügt, um Vanillinmandelsäure zu Vanillin umzusetzen. Es werden also durch Vanillinbildung hohe Reagenzleerwerte vorgetäuscht. Setzt man aber zuerst die Dithionitlösung und dann die Natrium-meta-perjodatLösung zu, so wird kein Vanillin gebildet. Bei Urinproben (auch mit $50 \mu \mathrm{g}$ Vanillinmandelsäure aufgestockt) waren bei der gleichen Einwirkungszeit die gemessenen Extinktionen der Leerwerte unabhängig von der Reihenfolge der Reagenzzugabe. Setzte man keine der beiden Lösungen zu, wurden die gleichen Extinktionen gemessen. Wir haben vorsichtshalber die angegebene Reihenfolge eingehalten.

Bei verdünnten Urinproben kann das oben angegebene Verfahren dahingehend abgeändert werden, daß beim letzten Schritt Toluol anstatt mit $2 \mathrm{ml}$ mit $1 \mathrm{~m} / 1 \mathrm{M} \mathrm{K}_{2} \mathrm{CO}_{3}$ ausgeschüttelt wird. Die Extinktion wird dann in einer MT-2-Küvette gemessen. Bei Parallelmessung von 8 verschiedenen Urinproben nach der oben angegebenen Vorschrift und dieser Anderung wurde kein signifikanter Unterschied $(P=0,01)$ festgestellt.

\section{Zuverlässigkeitskriterien der Methode} Präzision

Zur Präzisionskontrolle werden täglich jeweils zwei Proben eines Kontrollurins bei einer Untersuchungsserie mitgeführt. Die Kontrollutinproben wurden so hergestellt, da $\beta 10$ / Sammelurin mit etwa $100 \mathrm{ml} 6 \mathrm{~N} \mathrm{HCl}$ angesäuert und dann in Einzelportionen von etwa $20 \mathrm{ml}$ aufgeteilt wurden.

Die Urinproben werden bis zur. Analyse in einer Tiefkühltruhe bei etwa $-30^{\circ}$ aufbewahrt.

Für die Streuung von Tag zu Tag aus 48 Analysen an 48 Tagen betrug der Variationskoeffizient 4,8\%. Bei der Streuung in der 
Serie, berechnet aus 48 Doppelbestimmungen an 48 Tagen, betrug der Variationskoeffizient 1,6\%. Das arithmetische Mittel $\overline{\mathrm{x}}$ für die bestimmte Vanillinmandelsäure-Konzentration in den Kontrollproben betrug $3,6 \mathrm{mg} / \mathrm{l}$ Urin.

\section{Spezifität der Metbode}

Die Spektren von Vanillin in $1 \mathrm{M} \mathrm{K}_{2} \mathrm{CO}_{3}$, einer reinen Vanillinmandelsäure-Lösung, eines Poolurins und einer mit $10 \mu \mathrm{g}$ aufgestockten Poolurinprobe nach Umsatz mit Natrium-metaperjodat und Isolierung des Vanillins in der oben beschriebenen Weise wurden mit dem selbstregistrierenden Spektralphotometer DMR 21 (Zeiss) gemessen. Die Spektren des Vanillins und der oxydierten Vanillinmandelsäure einerseits und der Poolurinprobe sowie des aufgestockten Poolurins andererseits waren identisch. Gegenüber den ersteren, deren Absorptionsmaximum bei $345 \mathrm{~nm}$ lag, war bei den Urinproben das Maximum um etwa $5 \mathrm{~nm}$ zum Kurzwelligen verschoben. Diese Verschiebung wurde bei mehrfachen Kontrollen mit verschiedenen Urinproben gefunden. Sie dürfte auf den Einfluß des $p$-Hydroxybenzaldehyds (Absorptionsmaximum bei $330-335 \mathrm{~nm}$ ) zurückgehen, der bei dieser Methode aus der im Urin ausgeschiedenen p-Hydroxymandelsäure entsteht.

\section{Wiederauffindungsrate}

Zugabe von $10 \mu \mathrm{g}$ Vanillinmandelsäure $/ 5 \mathrm{~m} /$ Urin

$\begin{array}{ll}\overline{\mathrm{x}} & =9,3 \mu \mathrm{g} \\ \mathrm{n} & =12\end{array}$

$\mathrm{s} \quad=0,63 \mu \mathrm{g}$

$\Delta \overline{\mathrm{x}}(\mathrm{P}=0,05)= \pm 0,40 \mu \mathrm{g}$

Wiederauffindung in $\%=93 \pm 4,0$.

\section{Nacbueisgrenze}

Die Nachweisgrenze (17), berechnet aus 3 sI Grenze der Streuung der Leerwerte von 57 Urinproben unterschiedlicher Konzentration, lag bei $0,5 \mu \mathrm{g}$ Vanillinmandelsäure $/ \mathrm{m} l$ Urin $\left(\overline{\mathbf{x}}+3 \mathrm{~s}_{\mathrm{J}}\right)$.

\section{Ergebnisse und Diskussion}

Ermittlung der Reaktionsbedingungen bei der Oxydation

Menge des Oxydationsmittels: Die Menge an Natriummeta-perjodat-Lösung ist unkritisch, wie das Ergebnis folgender Versuche zeigt. $5 \mathrm{~m} l$ Poolurinproben wurden nach Vorschrift ausgeschüttelt. Je $1 \mathrm{ml}$ der $\mathrm{K}_{2} \mathrm{CO}_{3}$ Phase wurde mit $0,1,0,2,0,3,0,4$ und $0,5 \mathrm{ml} 2$ proz. Natrium-meta-perjodat-Lösung oxydiert. Die Menge an Reduktionsmittel wurde in gleicher Weise erhöht. Die übrigen Parameter wurden konstant gehalten. Die arithmetischen Mittel $(n=3)$ der gefundenen Vanillinmandelsäure-Konzentrationen lagen zwischen 20,2 und $21,7 \mu \mathrm{g} / 5 \mathrm{~m} l$ Urin.

Zuckereinfluß: Glucose reagiert mit Natrium-metaperjodat und verbraucht somit Oxydationsmittel. Beim Ausschütteln des angesäuerten Urins sollte zwar keine Glucose mitabgetrennt werden, aber bei der von uns benutzten Analysendurçhführung ist eine Verschleppung möglich. Deshalb wurden in Dreifachbestimmungen je $5 \mathrm{~m} l$ Poolurin ohne, mit $2,5 \mathrm{~g} / 100 \mathrm{ml}$ und $5,0 \mathrm{~g} / 100 \mathrm{~m} / \mathrm{Glucose}$ analysiert. Die Oxydation wurde mit 0,2 $\mathrm{m} l$ 2proz. Natrium-meta-perjodat-Lösung durchgeführt. Die bestimmten Vanillinmandelsäure-Konzentrationen lagen $z$ wischen 19,0 und $19,2 \mu \mathrm{g} / 5 \mathrm{~m} l$ Urin. Eine mögliche Störung durch zusätzlichen Verbrauch an Oxydationsmittel bei der Analyse von zuckerhaltigen Urinen konnte somit ausgeschlossen werden.
Reaktionstemperatur: Von BRUNJES und Mitarbeitern (18) wurde eine Erhöhung der Temperatur von $50^{\circ}$ auf $100^{\circ}$ und eine Verlängerung der Oxydationszeit von 30 auf $60 \mathrm{Min}$. vorgeschlagen. Andererseits war gezeigt worden, daß bei einer Oxydationsdauer von 30 Min. und einer Temperatur von $60^{\circ} 5 \%$ des Vanillins zerstört wurden (1). Diese widersprüchlichen Angaben waren der Anlaß, den Einfluß dieser Parameter nochmals zu überprüfen.

Tab. 1

Einfluß der Reaktionstemperatur auf reine Lösungen von Vanillin ohne Zusatz von NaJO, (1), mit Zusatz von NaJO, (2), beim Umsatz von reinen Losungen von Vanillinmandelsäure zu Vanillin (3), auf $10 \mu \mathrm{g}$ Vanillinmandelsäure (5). Die angegebenen Werte sind die arithmetischen Mittel von Dreifachbestimmungen. Werte sind die betrug $30 \mathrm{Min}$.

\begin{tabular}{|c|c|c|c|c|c|}
\hline \multirow{2}{*}{$\frac{\begin{array}{c}\text { Versuchs- } \\
\text { reihe }\end{array}}{1}$} & \multirow{2}{*}{$\frac{\underset{(\mu \mathrm{g})}{\text { zugesetzte Substanz }}}{15 \mu \mathrm{g} \text { Vanillin }}$} & \multicolumn{2}{|c|}{$\begin{array}{l}\text { Temperatur } 50^{\circ} \\
\text { Wiederfindung } \\
(\mu \mathrm{g}) \quad(\%)\end{array}$} & \multicolumn{2}{|c|}{$\begin{array}{l}\text { Temperatur } 70^{\circ} \\
\text { Wiederfindung } \\
(\mu \mathrm{g}) \quad(\%)\end{array}$} \\
\hline & & $\begin{array}{l}5,1 \\
9,9\end{array}$ & $\begin{array}{r}102 \\
99\end{array}$ & $\begin{array}{r}5,2 \\
10,1\end{array}$ & $\begin{array}{l}104 \\
101\end{array}$ \\
\hline 2 & $\begin{array}{r}5 \mu \mathrm{g} \text { Vanillin } \\
10 \mu \mathrm{g} \text { Vanillin }\end{array}$ & $\begin{array}{l}5,2 \\
9,7\end{array}$ & $\begin{array}{r}104 \\
97\end{array}$ & $\begin{array}{l}4,4 \\
9,0\end{array}$ & $\begin{array}{l}88 \\
90\end{array}$ \\
\hline \multirow[t]{2}{*}{3} & $5 \mu \mathrm{g}$ Vanillin- & 5,0 & 100 & 4,4 & 88 \\
\hline & $10 \mu \mathrm{g} \underset{\text { Vanillin- }}{\text { mandelsäure }}$ & 9,5 & 95 & 8,5 & 85 \\
\hline 4 & $5 \mathrm{ml}$ Poolurin & 17,1 & 一 & 17,2 & 一 \\
\hline 5 & $\begin{array}{l}5 \mathrm{ml} \text { Poolurin } \\
+10 \mu g \text { Vanillin- } \\
\text { mandelsäure }\end{array}$ & 26,1 & 90 & 26,5 & 93 \\
\hline
\end{tabular}

Zunächst wurden reine Lösungen von Vanillin ohne, mit Zusatz von $0,2 \mathrm{~m} l$ 2proz. Natrium-meta-perjodat und Vanillinmandelsäure mit Oxydationsmittelzusatz 30 Min. bei $50^{\circ}$ und $70^{\circ}$ erwärmt. Außerdem wurden Poolurine mit und ohne Aufstockzusatz in gleicher Weise analysiert (Tab. 1). Bei den reinen Lösungen nimmt die Ausbeute an wiedergefundenem Vanillin mit steigender Temperatur ab. Dies ist durch eine oxydative Zerstörung und nicht durch eine zunehmende Instabilität des Vanillins in alkalischer Lösung bei steigender Temperatur bedingt, wie ein Vergleich der Werte von Versuchsreihe 1 und 2 zeigt. Bei den Urinproben zeigt sich kein Einfluß der Oxydationstemperatur. Die Extinktionen der Proben, die bei $70^{\circ}$ oxydiert wurden, lagen zwar höher als die der bei $50^{\circ}$ oxydierten, aber auch die Extinktionen der Leerwerte waren erhöht.

Reaktionszeit: Bei dem folgenden Versuch wurden zwei verschiedene Poolurinproben mit und ohne Aufstockzusatz 30 und $60 \mathrm{Min}$. bei $50^{\circ}$ oxydiert (Tab. 2). Nach diesen Werten ist die Verdoppelung der Oxydationszeit bei $50^{\circ}$ ohne Einfluß.

Tab. 2

Vanillinmandelsäure-Bestimmung in zwei verschiedenen Poolurinproben ohne und mit Aufstockzusatz in Abhängigkeit von der Oxydationszeit. Die Oxydationstemperatur betrug $50^{\circ}$. Die Werte sind die arithmetischen Mittel aus Dreifachbestimmungen

\begin{tabular}{|c|c|c|c|c|}
\hline Probe & \multicolumn{2}{|c|}{$\begin{array}{c}30 \text { Min. } \\
\text { Oxydationszeit }\end{array}$} & \multicolumn{2}{|c|}{$\begin{array}{c}60 \mathrm{Min} . \\
\text { Oxydationszeit }\end{array}$} \\
\hline Poolurin & 16,7 & - & 16,6 & - \\
\hline aufgestockter Poolurin & 25,8 & 91 & 25,9 & 93 \\
\hline Poolurin & 18,7 & - & 19,1 & - \\
\hline aufgestockter Poolurin & 28,1 & 94 & 28,0 & 89 \\
\hline
\end{tabular}


Die in der Originalarbeit (1) angegebenen Versuchsbedingungen für die Oxydation von Vanillinmandelsäure zu Vanillin können auf Grund der bisher angeführten Versuchsergebnisse beibehalten werden. In der Arbeitsvorschrift ist allerdings die doppelte Menge an Oxydationsmittel angegeben, um mit dem Überschuß bei eventuell auftretendem größeren Verbrauch an Natrium-meta-perjodat durch Störsubstanzen eine gewisse Reserve zu haben.

\section{Abtrennung des Vanillins}

Nach PIsAno und Mitarbeitern (1) wird zur pH-Einstellung nach der Oxydation ein $3 \mathrm{~N}^{\mathrm{KH}_{2}} \mathrm{PO}_{4}$-Puffer pH 7,5 benutzt. Dieser kristallisiert beim Ansetzen leicht aus. Daher wurden verschiedene Puffer zum Einstellen des pH-Wertes der wäßr. Phase nach der Oxydation geprüft, indem der VanillinmandelsäureGehalt von Poolurinproben bestimmt wurde. Von jedem Puffer wurden $0,6 \mathrm{~m} l$ - bei Beibehaltung der in der Arbeitsvorschrift angegebenen Volumina der übrigen Reagenzien - zugesetzt. Die Toluolphase wurde mit $2,0 \mathrm{ml}$ des entsprechenden Puffers nachgewaschen. Für jeden Versuchsansatz wurden zwei Vanillinmandelsäure-Standards mitgeführt. Die $\mathrm{pH}$-Werte der wäßr. Phasen wurde elektrometrisch bestimmt. Das Ergebnis ist in Tabelle 3 wiedergegeben.

Tab. 3

Vanillinmandelsäure-Konzentration von Poolurinproben, wobei das gebildete Vanillin bei Benutzung verschiedener Puffer isoliert wurde. Die angegebenen Werte sind die arithmetischen Mittel aus Dreifachbestimmungen (Ansetzen der Pufferlösungen s. Reagenzien)

\begin{tabular}{|c|c|c|c|c|c|c|c|}
\hline \multicolumn{2}{|c|}{$\begin{array}{c}3 \mathrm{~N}_{\mathrm{KH}}^{\mathrm{K}} \mathrm{PO}_{4} / \\
\mathrm{NaOH} \\
\mathrm{pH} 7,5 \text { (1) }\end{array}$} & \multicolumn{2}{|c|}{ 2N $\begin{array}{c}\mathrm{KH}_{2} \mathrm{PO}_{4} / \\
\mathrm{NaOH} \\
\mathrm{pH} \mathrm{7,5}\end{array}$} & \multicolumn{2}{|c|}{$\begin{array}{c}2 \mathrm{~N} \mathrm{KH}_{2} \mathrm{KO}_{4} / \\
\mathrm{NaOH} \\
\mathrm{pH} 7,0\end{array}$} & \multicolumn{2}{|c|}{$\begin{array}{r}1,5 \mathrm{M} \mathrm{KH}_{2} \mathrm{PO} \\
1,0 \mathrm{M} \mathrm{K}_{2} \mathrm{HPO} \\
\mathrm{pH} 6,3(19)\end{array}$} \\
\hline $\begin{array}{c}\mu \mathrm{g} / 5 \mathrm{~m} l \\
19,6\end{array}$ & $\begin{array}{l}\mathrm{pH} \\
7,5\end{array}$ & $\begin{array}{c}\mu \mathrm{g} / 5 \mathrm{ml} \\
19,3\end{array}$ & $\begin{array}{l}\mathrm{pH} \\
7,5\end{array}$ & $\begin{array}{c}\mu \mathrm{g} / 5 \mathrm{ml} \\
19,5\end{array}$ & $\begin{array}{l}\mathrm{pH} \\
7,3\end{array}$ & $\begin{array}{c}\mu \mathrm{g} / 5 \mathrm{ml} \\
19,6\end{array}$ & pH \\
\hline
\end{tabular}

Das Ergebnis der Versuche zeigt, daß trotz unterschiedlichem $\mathrm{pH}$-Wert der wäßr. Lösung bei Verwendung verschiedener Puffer kein Unterschied der gemessenen Vanillinmandelsäure-Werte auftritt. Die Extinktionen der Standards waren bei den verschiedenen Ausschüttelbedingungen gleich. Für die Arbeitsvorschrift wurde der $2 \mathrm{~N} \mathrm{KH}_{2} \mathrm{PO}_{4}$-Puffer $\mathrm{pH} 7,5$ ausgewählt.

\section{Auswertung}

Standard: Wie im methodischen Teil erwähnt; erfolgt die Berechnung des Vanillinmandelsäure-Gehaltes der Probe über einen wäßr. Standard, der durch den ganzen Analysengang mitgeführt wird (Standard A). Benutzt man einen Standard, der erst bei der Oxydation eingesetzt wird (Standard B), so liegen die bestimmten Vanillinmandelsäure-Werte niedriger (Tab. 4). Der Verlust vom Ausschütteln des angesäuerten Urins bis zur Oxydation liegt nach diesen Werten zwischen 12 und 15\%. Dies stimmt mit den Literatur-Angaben etwa überein, wonach bei einmaligem Ausschütteln mit Äthylacetat $90 \%$ der Vanillinmandelsäure abgetrennt werden.

Tab. 4

Vanillinmandelsäure-Bestimmung in Poolurinproben, ohne und mit $10 \mu \mathrm{g}$ Vanillinmandelsäure aufgestockt, bei Auswertung über verschiedene Standards. Standard $A=$ Vanillinmandelsäure-Standard, der durch den ganzen Analysengang mitgeführt wird. Standard $\mathbf{B}=$ Vanillinmandelsäure-Standard, der von der Oxydation an mitgeführt wird $(+=$ bezogen auf die Wiederfindung)

\begin{tabular}{|c|c|c|c|c|c|c|}
\hline Probe & Standard & $\underset{(\mu \mathrm{g})}{\dot{\overline{\mathbf{x}}}}$ & $\mathbf{s}$ & $\mathrm{n}$ & $\begin{array}{l}\text { Wieder- } \\
\text { findung } \\
(\%)\end{array}$ & $\begin{array}{c}V \\
(\%)\end{array}$ \\
\hline Poolurin & $\begin{array}{l}\mathrm{A} \\
\mathrm{B}\end{array}$ & $\begin{array}{l}19,2 \\
16,4 .\end{array}$ & $\begin{array}{l}0,44 \\
0,85\end{array}$ & $\begin{array}{r}12 \\
9\end{array}$ & 二 & $\begin{array}{l}2,3 \\
5,2\end{array}$ \\
\hline $\begin{array}{l}\text { Poolurin mit } \\
10 \mu \text { g Vanillin- } \\
\text { mandelsäure } \\
\text { aufgestockt }\end{array}$ & $\begin{array}{l}\text { A } \\
\text { B }\end{array}$ & $\begin{array}{l}28,6 \\
24,4\end{array}$ & $\begin{array}{l}0,63+ \\
0,41+\end{array}$ & $\begin{array}{r}12 \\
9\end{array}$ & $\begin{array}{l}93 \\
80\end{array}$ & $\begin{array}{l}6,7+ \\
5,3^{+}\end{array}$ \\
\hline
\end{tabular}

Leerwerteinfluß: Nach PISANo und Mitarbeitern (1) ist nur bei genauen Untersuchungen eine Bestimmung des Urinleerwertes erforderlich. Bei einer Modifikation der Methode (19) betrug die Extinktion des Urinleerwertes etwa 30\% der Extinktion der Proben. Wir haben diese Frage noch einmal aufgegriffen, um zu klären, ob der Leerwerteinfluß konstant additiv oder proportional der Konzentration ist. Die 24-Stunden-Urine des Normalkollektivs und die 3-Stunden-Urinproben eines Vorversuchs zur Untersuchung circadianer Rhythmen wurden mit Urinleerwerten analysiert. Die Vanillinmandelsäure-Gehalte wurden mit und ohne Berücksichtigung des Leerwertes berechnet. Die Extinktion des Reagenzleerwertes lag unter 0,01 und wurde beim Standard deshalb weggelassen.

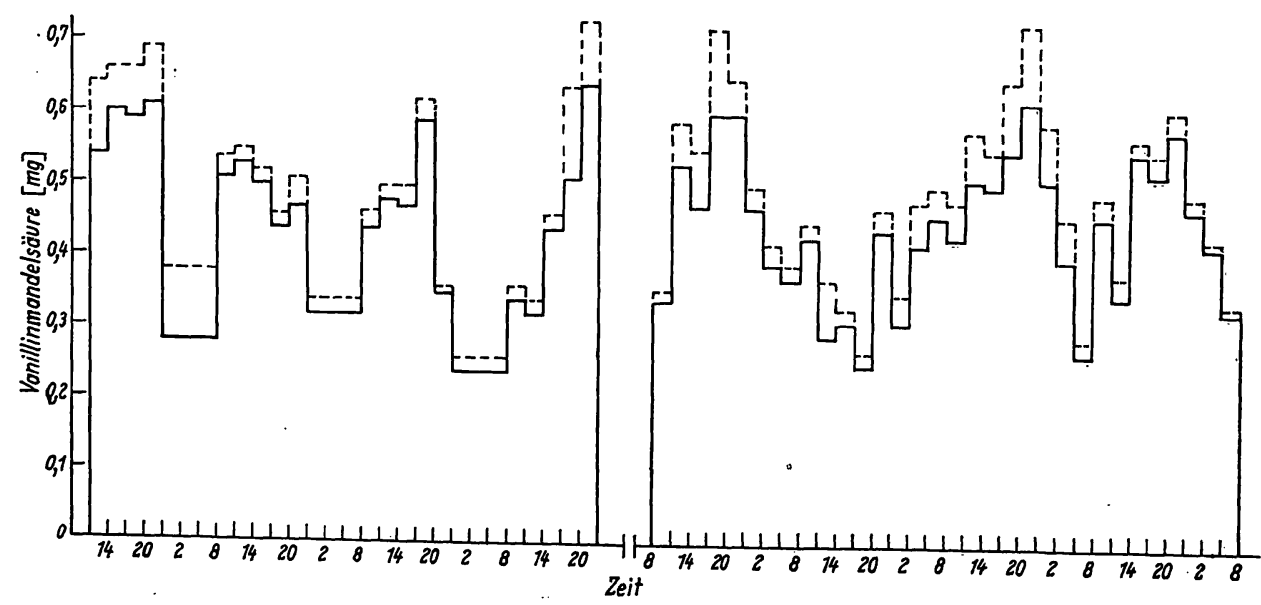

Abb. 1

Die tageszeitliche Änderung der Vanillinmandelsäure-Ausscheidung im Urin einer Versuchsperson unter verschiedenen Bedingungen. Die Sammelperioden betrugen 3 bzw. 9 Stunden. Die ausgeschiedenen Vanillinmandelsäure-Mengen wurden mit (durchzogene Linie) und ohne Berücksichtigung der Urinleerwerte (gestrichelte Linie) berechnet 
Das Ergebnis der Vanillinmandelsäure-Bestimmung in 3-Stunden-Urinen ist in Abbildung 1 wiedergegeben. Bei dieser Versuchsreihe mit normalem Licht-DunkelWechsel konnte die Versuchsperson während der ersten vier Versuchstage nachts durchschlafen, während sie in den darauffolgenden 4 Tagen nachts in dreistündigem Abstand geweckt wurde. Hier interessiert allerdings nur die analytische Frage, ob bei Nichtberücksichtigung der Urinleerwerte die gleichen quantitativen Aussagen möglich sind. Wie die Abbildung 1 zeigt, liefert die ohne Berücksichtigung der Leerwerte durchgeführte Vanillinmandelsäure-Bestimmung systematisch $\mathrm{zu}$ hohe Werte. Die größten Abweichungen können bei Proben niederer Konzentration auftreten. Bei der von uns gewählten Versuchsanordnung zeigt das Urinvolumen keine Tagesperiodik. Daraus folgt, da $\beta$ die Probe jeder Sammelperiode nieder konzentriert sein kann und dann eine größere Abweichung zeigt. Bei einer großen Anzahl von Analysen verteilen sich diese Fehler aber statistisch, so daß die Schwingungsbreiten der Zeitkurven nicht systematisch beeinflußt werden.

In Abbildung 2 sind die Ergebnisse der Analyse der 3und 24-Stunden-Urinproben zusammengefaßt. Aufgetragen ist die gemessene Vanillinmandelsäure-Konzentration ohne Berücksichtigung der Urinleerwerte gegen die Vanillinmandelsäure-Konzentration mit deren Berücksichtigung. Wie Abbildung 2 zeigt, sind beide Größen streng korreliert. Der Korrelationskoeffizient beträgt für die 3-Stunden-Urinwerte 0,994 und für die 24-Stunden-Urinwerte 0,996. Die Berechnung der Regressionsgeraden ergab folgendes Ergebnis:

$$
\begin{gathered}
\mathrm{y}_{1}=1,04 \mathrm{x}_{1}+0,1 \text { (3-Stunden-Urinwerte) } \\
\cdot \mathrm{y}_{2}=1,02 \mathrm{x}_{2}+0,2 \text { (24-Stunden-Urinwerte) }
\end{gathered}
$$

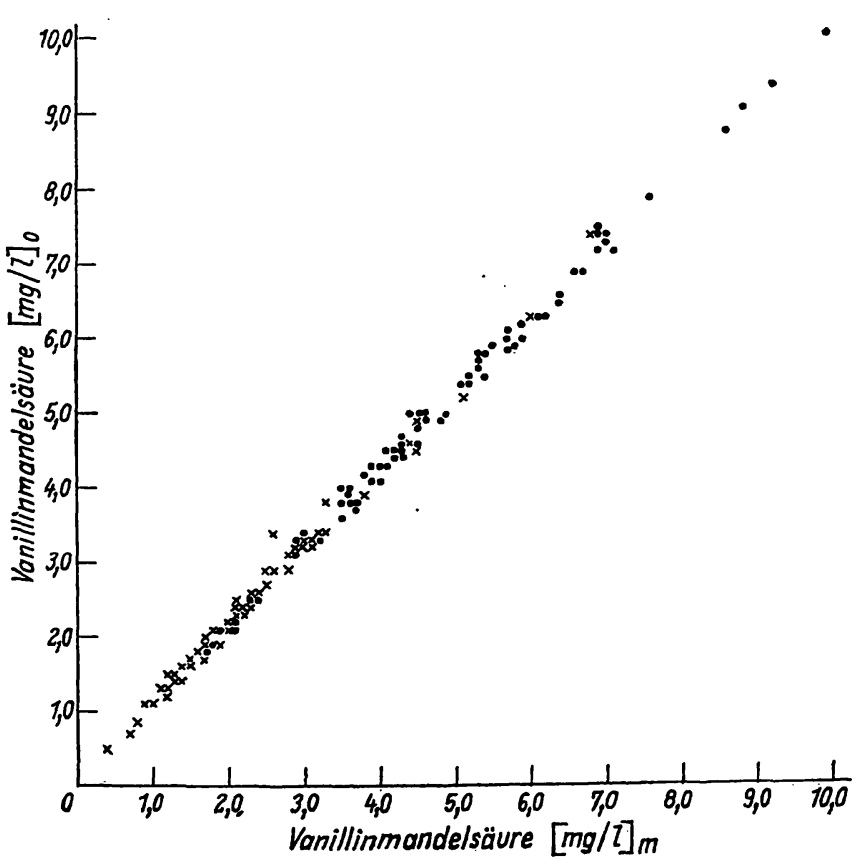

Abb. 2

Die Vanillinmandelsäure-Konzentration von 3 Stunden-Urinproben (Kreuze, $n=58$ ) und 24-Stunden-Urinproben (Punkte, $n=98$ ) ohne Berücksichtigung (Index 0) der Urinleerwerte gegen die Konzentration mit deren Berücksichtigung (Index m) aufgetragen.
Nach diesen Werten kann der Leerwerteinfluß sowohl konstant additiv wie auch konzentrationsabhängig sein. Zusammenfassend kann man sagen, daß sowohl bei der Bestimmung der 24-Stunden-Ausscheidung wie auch bei den Untersuchungen zur circadianen Rhythmik in 3-Stunden-Urinproben der Urinleerwert nicht mitbestimmt werden muß. Die systematische Abweichung liegt durchschnittlich unter 10\%. Die Arbeitsersparnis ist recht erheblich, da bei Weglassen der Urinleerwerte pro Tag die doppelte Anzahl von Proben analysiert werden kann.

\section{Normalwerte}

Es wurde die Vanillinmandelsäure-Ausscheidung in 24-Stunden-Urinen bei 47 Frauen und 51 Männern im Alter zwischen 18 und 65 Jahren, die ihrer normalen Tätigkeit nachgingen, bestimmt. Der Verzehr von Bananen, vanillinhaltigen Speisen, Tonic water und die Einnahme von Medikamenten waren 3 Tage vor Sammelbeginn untersagt ${ }^{5}$ ). Während des Sammelns mußten die Proben kühl aufbewahrt werden (Eisschrank, Kühltasche). Aus versuchstechnischen Gründen wurden die Urine erst nach der Sammelperiode mit $6 \mathrm{~N} \mathrm{HCl}$ angesäuert.

Die Meßwerte sind in Abbildung 3 nach Alter und Geschlecht zusammengestellt, und zwar ohne und mit Berücksichtigung der Urinleerwerte berechnet. Es konnte keine statistisch signifikante Alters- und Geschlechtsabhängigkeit festgestellt werden $(P=0,05)$.

Die Werte des von uns ermittelten Normalbereiches für die Vanillinmandelsäure-Ausscheidung in 24-StundenUrinen sind in Tabelle 5 zusammengefaßt. Als Kenn-

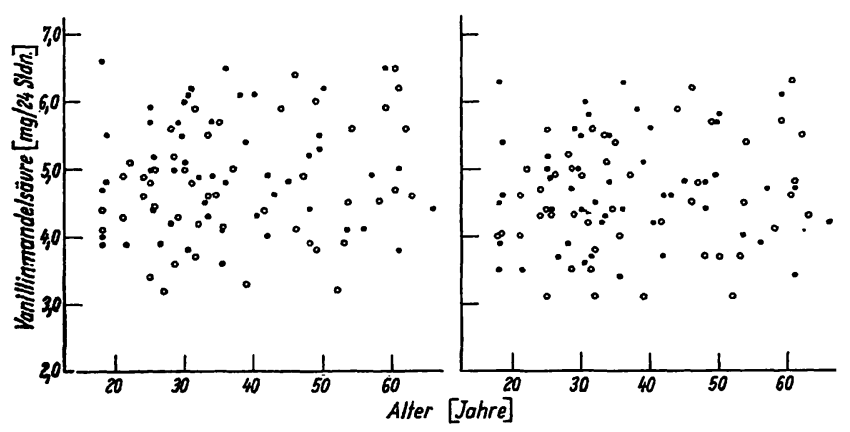

Abb. 3

Die Werte der 24-Stunden-Ausscheidung der Vanillinmandelsäure im Urin. Links ohne Berücksichtigung und rechts mit Berücksichtigung der Urinleerwerte berechnet. Punkte $=$ Männer, $\mathrm{n}=51$; Kreise $=$ Frauen $(n=47)$

Tab. 5

Normalbereich der Vanillinmandelsäure-Ausscheidung in 24-StundenUrinen, wobei die Einzelwerte mit und ohne Berücksichtigung der Urinleerwerte für 51 Männer und 47 Frauen ermittelt wurden

\begin{tabular}{llcc}
\hline & Kollektiv & Median & 96 Percentil \\
\hline Männer & ohne Urinleerwert & 4,9 & $3,8-6,5$ \\
$\mathrm{n}=51$ & mit Urinleerwert & 4,6 & $3,4-6,3$ \\
Frauen & ohne Urinleerwert & 4,6 & $3,2-6,4$ \\
n $=47$ & mit Urinleerwert & 4,4 & $3,1-6,2$ \\
Gesamt & ohne Urinleerwert & 4,8 & $3,3-6,5$ \\
n $=98$ & mit Urinleerwert & 4,6 & $3,1-6,3$ \\
\hline
\end{tabular}

5) Bestimmte Einschränkungen waren crforderlich, da auch noch andere Bestandteilc bestimmt wurden. 
größen wurden Median und 96 Percentil gewählt, um unabhängig zu sein von dem theoretischen Modell, das auf die Verteilung dieser Werte angewandt werden kann.

Zum Vergleich sind in Tabelle 6 die in der Literatur angegebenen Normalwerte, Größe der Kollektive, benutzte Methode sowie Literaturzitat zusammengefaßt. Es besteht eine gute Übereinstimmung des von uns ermittelten Normalbereichs mit den Werten von v. STUDNitz (9). Letztere wurden mit einer anderen Methode ermittelt. Die mit fast der gleichen Methọde (1, 19), ebenso wie die mit der „Kaliumferricyanidmethode" ermittelten Normalwerte (10) zeigen gegenüber den von uns bestimmten erhebliche Abweichungen, insbesondere in bezug auf die untere Grenze.

Tab. 6

Literaturübersicht: Die Vanillinmandelsäure-Ausscheidung in 24Stunden-Urinen mit verschiedenen Methoden bestimmt

\begin{tabular}{|c|c|c|c|c|c|}
\hline $\begin{array}{l}\text { Normal- } \\
\text { bereich } \\
\text { mg/24 Stdn. }\end{array}$ & 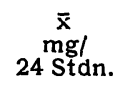 & $\mathrm{n}$ & Besonderheiten & Methode & Lit. \\
\hline $3,2-6,4$ & $\begin{array}{l}4,81 \\
4,97\end{array}$ & 62 & $\begin{array}{l}\text { Frauen } \\
\text { Männer }\end{array}$ & $\begin{array}{l}\text { Hochspan- } \\
\text { nungselek- } \\
\text { trophorese }\end{array}$ & $\stackrel{9}{9}(1960)$ \\
\hline $\begin{array}{l}0,7-6,8 \\
0,5-8,6\end{array}$ & $\begin{array}{l}4,0 \\
4,2\end{array}$ & $\begin{array}{r}68 \\
103\end{array}$ & $\begin{array}{l}\text { gesunde Vp. } \\
\text { Hypertoniker }\end{array}$ & $\begin{array}{l}\mathrm{K}_{3}\left[\mathrm{Fe}(\mathrm{CN})_{6}\right] \\
\text { Oxydation }\end{array}$ & $\begin{array}{c}10 \\
(1960)\end{array}$ \\
\hline $\begin{array}{l}1,73-3,74 \\
2,58-4,56\end{array}$ & $\begin{array}{l}2,91 \\
3,68\end{array}$ & $\begin{array}{l}14 \\
10\end{array}$ & $\begin{array}{l}\text { O' Krankenhaus- } \\
\text { patienten }\end{array}$ & $\begin{array}{l}\text { Isotopen- } \\
\text { verdünnung }\end{array}$ & $\begin{array}{c}8 \\
(1961)\end{array}$ \\
\hline $1,5-5,9$ & 3,7 & 20 & $\begin{array}{l}\text { Pat. mit primä- } \\
\text { rer Hypertonie }\end{array}$ & $\begin{array}{l}\mathrm{NaJO}_{\text {Oxydation }} \\
\text { Oxyd }\end{array}$ & $\begin{array}{c}1 \\
(1962)\end{array}$ \\
\hline $1,7-7,4$ & 4,1 & 361 & $\begin{array}{l}\text { Screening-Test } \\
\text { auf Phäochromo- } \\
\text { cytom }\end{array}$ & $\begin{array}{l}\mathrm{NaJO}_{4}^{-} \\
\text {Oxydation }\end{array}$ & $\begin{array}{c}19 \\
(1964)\end{array}$ \\
\hline $0-6,51$ & 3,27 & 190 & $\begin{array}{l}\text { Krankenhaus- } \\
\text { patienten }\end{array}$ & $\begin{array}{l}\text { Ionenaus- } \\
\text { tauscher } \\
p \text {-Nitroanilin }\end{array}$ & $\begin{array}{c}7 \\
(1965)\end{array}$ \\
\hline
\end{tabular}

\section{Diagnostischer Wert}

Die vergleichsweise einfachere Bestimmung der Vanillinmandelsäure wird häufiger als die Adrenalin- und Noradrenalin-Bestimmung bei der Phäochromocytomdiagnostik durchgeführt. Man sollte aber berücksichsichtigen, daß die Vanillinmandelsäure-Bestimmung zu einer falsch negativen Beurteilung führen kann. CROUT, Pisano und Sjozrdsma (20) zeigen dies in einer $\mathrm{Zu}-$ sammenfassung von 23 Patienten mit einem gesicherten Phäochromocytom. Von diesen hatten 20 Patienten eine erhöhte Ausscheidung der Katecholamine, Metanephrine und der Vanillinmandelsäure. Bei den restlichen drei war die Ausscheidung der freien Katecholamine gering erhöht bei normalen "Metanephrin"- und Vanillinmandelsäure-Werten. K. ENGELMAN (21) berichtet, daß bei 62 Patienten mit einem Phäochromocytom in drei Fällen eine normale oder fast normale Ausscheidung der Vanillinmandelsäure, bei zwei Patienten der Metanephrine und ebenfalls in zwei Fällen der Katecholamine gefunden wurde. Auf Grund seiner Erfahrungen kommt er zu dem Schluß, daß bei den meisten Patienten eine einmalige Bestimmung der Vanillinmandelsäure oder der Metanephrine ausreicht, um die Diagnose zu sichern. Erwähnt sei, daß bei Tumoren der Neuroblasten oder Sympathocyten in erster Linie die methoxylierten Derivate von Dopa, Dopamin und Noradrenalin vermehrt im Urin ausgeschieden werden (22).

Fräulein J.-M. QUANTE danken wir für ihre sorgfältige Mitarbeit und ihre Einsatzbereitschaft.

\section{Literatur}

1. Pisano, J. J., J. R. Crout und D. Abraham, Clin. Chim. Acta, Amsterdam 7, 285 (1962). - 2. Armstrong, M. D., K. N. F. Shaw und P. E. Wall, J. biol. Chemistry 218, 293 (1956). 3. Armstrong, M. D., A. McMillan und K. N. F. Shaw, Biochim. biophysica Acta, Amsterdam 25, 422 (1957). - 4. Schmid, E. und N. Henning, Klin. Wschr. 41, 566 (1963). - 5. Segura-Cardona, R. und K. Soenring, Med. exp. 10, 251 (1964). - 6. TaUtz, N. A., G. VoltMer und E. Schmid, Klin. Wschr. 43, 233 (1963). 7. Mrils, G. C., Texas Rep. Biol. Med. 23, 753 (1965). - 8. WEISE, V. K., R. K. McDonald und E. H. Labrosse, Clin. Chim. Acta, Amsterdam 6, 79 (1961). - 9. v. Studnitz, W., Scand. J. clin. Laborat. Invest. 12, Suppl. 48 (1960). - 10. Sunderman, F. W. jt., P. D. Clevveland, N. C. Law und F. W. Sunderman, Amer. J. Clin. Path. 34, 293 (1960). - 11. Weil-Malmerbe, H., Analytic. Biochem. 7, 485 (1964). - 12. Thomas, H. und W. DirscherL,
Arzneimittel-Forsch., Aulendorf 12, 429 (1962). - 13. SANDLER, M. und C. R. J. RuthVEN, Biochem. J. 80, 78 (1961). - 14. Rosano, C. L., Clin. Chem., New York 10, 673 (1964). - 15. Wilk, Sh., St. E. Gitlow, M. Mendlowitz, M. J. Franklin und H. E. CARR, Analytic. Biochem. 13, 544 (1965). - 16. WeILMalmerbe, H., Meth. Biochem. Analysis 16, 319 (1968). 17. KaIser, H., Z. analyt. Chem. 209, 1 (1965). - 18. BRunJes, S., D. Wybenga, J. Sproed und A. L. Chaney, Clin. Chem., New York 8, 452 (1962). - 19. Connelian, J. P. und J. M. Godfrex, Clin. Chim. Acta, Amsterdam 9, 410 (1964). - 20. CRout, J. R., J. J. Pisano und A. SJoerdsma, Am. Heatt J. 61, 375 (1961). 21. Sjoerdsma, A., K. Engelman, Th. A. Waldmann, L. H. Cooperman und W. G. Hammond, Ann. Int. Med. 65, 1302 (1966). - 22. Gjessing, L. R., Adv. Clin. Chemistry 11, 81 (1968).
Dr. Dr. H. Wisser PD. Dr. Dr. D. Stamm 8000 München 23 Kraepelinstr. 10 